\title{
Domain Walls in Supersymmetric QCD: From Weak to Strong Coupling
}

\author{
B. de Carlos* \\ Theory Division, CERN, CH-1211 Geneva 23, Switzerland \\ J. M. Moreno ${ }^{\dagger}$ \\ Instituto de Estructura de la Materia, CSIC, Serrano 123, 28006 Madrid, Spain
}

(Received 25 May 1999)

\begin{abstract}
We consider domain walls that appear in supersymmetric QCD with $N_{f}<N_{c}$ massive flavors. In particular, for $2 N_{f}<N_{c}$ we explicitly construct the domain walls that interpolate between vacua labeled by $i$ and $i+N_{f}$. We show that these solutions are Bogomol'nyi-Prasad-Sommerfield saturated for any value of the mass of the matter fields. This fact allows us to evaluate the large mass limit of these domain walls. We comment on the relevance of these solutions for supersymmetric gluodynamics.
\end{abstract}

PACS numbers: 11.30.Pb, 11.27.+d, 11.30.Er

In recent times, a lot of attention has been drawn to the existence of exact solutions in supersymmetric gauge theories that are in the strong coupling regime. One of the more relevant issues is that of domain walls in $\mathrm{SU}(N)$ supersymmetric gluodynamics, the theory of gluons and gluinos. Those arise because this theory has an axial U(1) symmetry broken by the anomaly to a discrete $Z_{2 N}$ chiral symmetry. Because of nonperturbative effects gluino condensates $(\langle\lambda \lambda\rangle)$ form, breaking the symmetry further down to $Z_{2}$. This leaves us with a set of $N$ different vacua labeled by

$$
\langle\operatorname{Tr} \lambda \lambda\rangle=\Lambda^{3} e^{2 \pi i k / N} \quad k=0,1, \ldots, N-1,
$$

where $\Lambda$ is the condensation scale, and, as indicated above, a set of domain walls interpolating between them. (In Ref. [1] it was pointed out the existence of a chirally symmetric vacuum where the gaugino condensate vanishes. Here we will consider only domain walls involving chirally asymmetric vacua.) If we assume that they are Bogomol'nyi-Prasad-Sommerfield (BPS) saturated, the energy density of these walls is exactly calculable and given by [2-5]

$$
\epsilon=\frac{N}{8 \pi^{2}}\left|\langle\operatorname{Tr} \lambda \lambda\rangle_{\infty}-\langle\operatorname{Tr} \lambda \lambda\rangle_{-\infty}\right|
$$

In fact, it has been suggested in Ref. [6] that, in the large $N$ limit, these domain walls are BPS states. On the other hand, these solutions preserving half of the supersymmetry would play an important role in the D-brane description of $N=1$ supersymmetric QCD (SQCD) [7]. However, we want to stress that whether or not these configurations were BPS saturated was, up to now, still an open question [5].

A useful way of gaining intuition on pure gluodynamics is by adding matter fields and analyzing the limit where these extra fields become very heavy. These new fields are usually taken to be pairs of chiral superfields transforming as $(N, \bar{N})$ under the color group. In the strong coupling regime, squark condensates will form. These models, for the case of $(N-1)$ flavors, were considered in Refs. [8-11], where the analysis of the vacuum struc- ture led the authors to conclude that the existence of BPS saturated domain walls was restricted to values for the mass $m$ of the squark fields below a certain critical one. This jeopardized the idea of recovering pure gluodynamics by taking the limit $m \rightarrow \infty$, which is precisely the issue we want to revise here.

In order to do that let us consider supersymmetric QCD with $\mathrm{SU}\left(N_{c}\right)$ gauge group and $N_{f}$ couples of chiral superfields $\left(Q_{i}, \bar{Q}_{i}\right)$ transforming as $N_{c}, \bar{N}_{c}$. Nonperturbative effects become relevant at the scale $\Lambda$, where condensates form. The gaugino and squark colorless condensates are described by the following composite fields:

$$
\begin{aligned}
S & =\frac{3}{32 \pi^{2}} \operatorname{Tr} W^{2}, \\
M_{j}^{i} & =Q^{i} \bar{Q}_{j} \quad i, j=1,2, \ldots, N_{f},
\end{aligned}
$$

where $W^{2}$ is the composite chiral superfield whose lowest component is $\lambda \lambda$. In this regime, the relevant degrees of freedom are described by a Wess-Zumino model, as shown in Ref. [12]. Its effective Lagrangian is given by

$$
\mathcal{L}=\frac{1}{4} \int d^{4} \theta \mathcal{K}+\frac{1}{2}\left[\int d^{2} \theta \mathcal{W}+\text { H.c. }\right],
$$

where $\mathcal{K}$ is the Kähler potential and $\mathcal{W}$ is the superpotential

$$
\mathcal{W}=\frac{2}{3} S \ln \frac{S^{N_{c}-N_{f}} \operatorname{det} M}{\Lambda^{3 N_{c}-N_{f}} e^{N_{c}-N_{f}}}-\frac{1}{2} \operatorname{Tr}(m M),
$$

with $m_{k}^{j}$ the mass matrix for the matter superfields. We will work in the flavor basis where this matrix is diagonal, and will use the notation $m_{k}^{j}=\delta_{k}^{j} m_{j}$. We will analyze the simple case where the corresponding eigenvalues are real. This superpotential has $N_{c}$ extrema labeled by the different phases of the gaugino condensate. At the minimum we have the gaugino condensate fixed to

$$
S_{*}^{N_{c}}=\left(\frac{3}{4}\right)^{N_{f}} \operatorname{det} m
$$


where, and from now on, we set $\Lambda=1$. The matter fields are aligned with respect to the former and given by

$$
M_{i *}^{j}=\delta_{i}^{j} \frac{1}{m_{i}} \frac{4}{3} S_{*} .
$$

Finally, the superpotential at the minimum is proportional to the gaugino condensate

$$
\mathcal{W}_{*}=-\frac{2}{3} N_{c} S_{*} .
$$

We want to study domain wall configurations that interpolate between the different minima. Here a technical problem appears: the superpotential has several branches associated with its logarithmic piece [13]. In the pure supersymmetric gluodynamics limit described by Veneziano and Yankielowicz [12] this is a severe problem, since any configuration going from one vacuum to another has to cross this branch. This is not necessarily the case when we include other fields, given that the variation in the phase of the gaugino condensate can be partially compensated by these new fields. In this case, this will be done by matter fields.

Let $(S, M)_{a}$ be a particular vacuum. We can continuously deform it into another vacuum, $(S, M)_{b}$. For this path in the configuration space, we define $\delta, w_{i}$ such that

$$
\begin{aligned}
\left.S\right|_{b} & =\left.e^{i \delta} S\right|_{a}, \\
\left.M_{i}^{i}\right|_{b} & =\left.e^{i\left(\delta+2 \pi w_{i}\right)} M_{i}^{i}\right|_{a}, \quad i=1, \ldots, N_{f} .
\end{aligned}
$$

Since, as mentioned above, matter at the minimum has to be aligned with respect to the gaugino condensate, $w_{i}$ must be some integer numbers. On the other hand, one necessary condition to avoid crossing the logarithmic branch along this general path is

$$
\left(N_{c}-N_{f}\right) \delta+\sum_{i}\left(\delta+2 \pi w_{i}\right)=0,
$$

and then $\delta=2 \pi \frac{k}{N_{c}}$, where $k$ is the integer given by $k=-\sum_{i} w_{i}$.

If we assume that there is a BPS domain wall connecting these two vacua from $z=-\infty$ to $z=+\infty$, it will be described by the following differential equations:

$$
\begin{aligned}
\mathcal{K}_{S \bar{S}} \partial_{z} \bar{S} & =e^{i \gamma} \frac{\partial \mathcal{W}}{\partial S}, \\
\mathcal{K}_{M \bar{M}} \partial_{z} \bar{M}_{i}^{i} & =e^{i \gamma} \frac{\partial \mathcal{W}}{\partial M_{i}^{i}},
\end{aligned}
$$

where $\mathcal{K}_{\phi \bar{\phi}}=\frac{\delta^{2} \mathcal{K}}{\delta \phi \delta \bar{\phi}}$ is the induced metric from the Kähler potential $\mathcal{K}$, and $\gamma$ is given by

$$
\gamma=-\frac{1}{2}(\delta+\pi)=-\frac{k \pi}{N_{c}}-\frac{\pi}{2} .
$$

Let us analyze the simplest case where the masses $m_{i}$ are degenerate. We will assume symmetric boundary conditions for the matter fields. To be more precise, we will consider $w_{i}=-1$ (and therefore $k=N_{f}$ ) for the path drawn by the domain wall. We can then assume that all the matter condensates have the same $z$ dependence. Then the configuration is described by four real functions

$$
\begin{aligned}
M_{i}^{i}(z) & =\left|M_{*}\right| \rho(z) e^{i \alpha(z)}, \\
S(z) & =\left|S_{*}\right| R(z) e^{i \beta(z)} .
\end{aligned}
$$

Notice that we have defined $\rho(z), R(z)$ in such a way that $\rho( \pm \infty)=R( \pm \infty)=1$. On the other hand, $\alpha$ varies from 0 to $2 \pi\left(N_{f} / N_{c}-1\right)$ and $\beta$ from 0 to $2 \pi N_{f} / N_{c}$. A consistent ansatz under reflection $z \rightarrow-z$ is given by $\rho(z)=\rho(-z), R(z)=R(-z), \beta(z)=2 \pi \frac{N_{f}}{N_{c}}-\beta(-z)$, and $\alpha(z)=2 \pi\left(\frac{N_{f}}{N_{c}}-1\right)-\alpha(-z)$. Then, we have the following boundary conditions at $z=0$ :

$$
\alpha(0)=\pi\left(\frac{N_{f}}{N_{c}}-1\right), \quad \beta(0)=\pi \frac{N_{f}}{N_{c}} .
$$

Equations (11) imply the following BPS constraint:

$$
\operatorname{Im}\left[e^{i \gamma} \mathcal{W}\left(S, M_{i}^{i}\right)\right]=\text { const . }
$$

In particular, at $z=0$ we have

$$
-R_{0}\left[\left(N_{c}-N_{f}\right)\left(\ln R_{0}-1\right)+N_{f} \ln \rho_{0}\right]-N_{f} \rho_{0}=N_{c} \cos \left(\pi \frac{N_{f}}{N_{c}}\right),
$$

where $R_{0}=R(0)$ and $\rho_{0}=\rho(0)$.

The case with $N_{f}=N_{c}-1$ has already been considered for $\mathrm{SU}(2)$ [8,9], $\mathrm{SU}(3)$ [10], and generalized to arbitrary $\mathrm{SU}(N)$ in $[11,14]$. Since $k=N_{c}-1$, the corresponding domain walls connect a minimun and its nearest neighbor. In these papers it was shown that these domain walls are BPS states only for squark masses lower than some critical value, $m_{*}$, that depends on $N_{c}$ and the Kähler potential. The existence of this bound is related to the presence of two different BPS domain wall solutions for small enough values of $m$, which became identical at the critical value.

We have done a similar analysis for other values of $N_{f}$, using the same Kähler potential, i.e., $\mathcal{K}=(S \bar{S})^{1 / 3}+$
$(M \bar{M})^{1 / 2}$. Here we have worked in detail the case $N_{c}=3, N_{f}=1$, whereas other cases will be presented elsewhere [15]. We have found that the equations can be solved for all values of the squark mass, and we have checked that the logarithmic branch is never crossed. The profiles for $\rho$ and $R$ are shown in Figs. 1 and 2 for several values of $m$ (given in units of $\Lambda$ ), focusing on their central region. The spatial coordinate $z$ is expressed in units of $\tilde{\Lambda}^{-1}$, where $\tilde{\Lambda}=\Lambda\left(\frac{3 m}{4 \Lambda}\right)^{N_{f} / 3 N_{c}}$ is the effective QCD scale that arises in the large $m$ limit.

In our case there is only one BPS solution for every value of $m$. This can be understood analyzing both the large and small $m$ limit. 


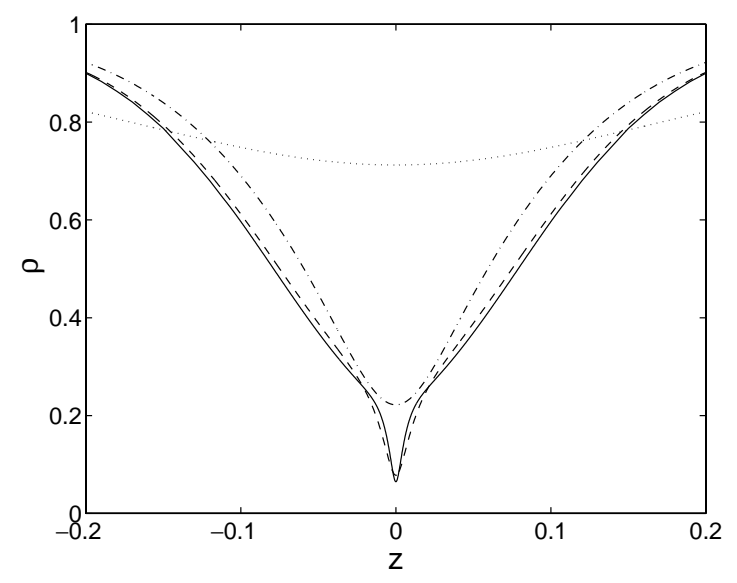

FIG. 1. $\rho(z)$ as defined in Eqs. (13) versus $z$ (in units of $\left.\tilde{\Lambda}^{-1}\right)$, for $m=2$ (dotted line), 20 (dash-dotted), 100 (dashed), and 200 (solid).

(i) When $m \ll \Lambda$ and $K_{S} \bar{S}$ is nonsingular, we can integrate out the gaugino condensate by imposing $\partial \mathcal{W} / \partial S=$ 0 . The corresponding Wess-Zumino model describing the matter condensate has a BPS state with the required boundary conditions for all values of $N_{f}<N_{c}$. In the case analyzed by Smilga and Veselov [10,11], there is yet another BPS solution that cannot be described by integrating $S$ out and that corresponds to $S \sim 0$. The existence of this domain wall is probably related to the fact that the Kähler metric $K_{S \bar{S}}$ is singular at this value [16]. In this limit $S \sim 0, \rho_{0}$ can be derived from Eq. (16), and it is given by

$$
\rho_{0}=-\frac{N_{c}}{N_{f}} \cos \left(\pi \frac{N_{f}}{N_{c}}\right) .
$$

In our case, since $N_{f} / N_{c}<1 / 2$, the resulting value for $\rho_{0}$ is never positive and therefore we do not find a second branch of solutions to the BPS equations.

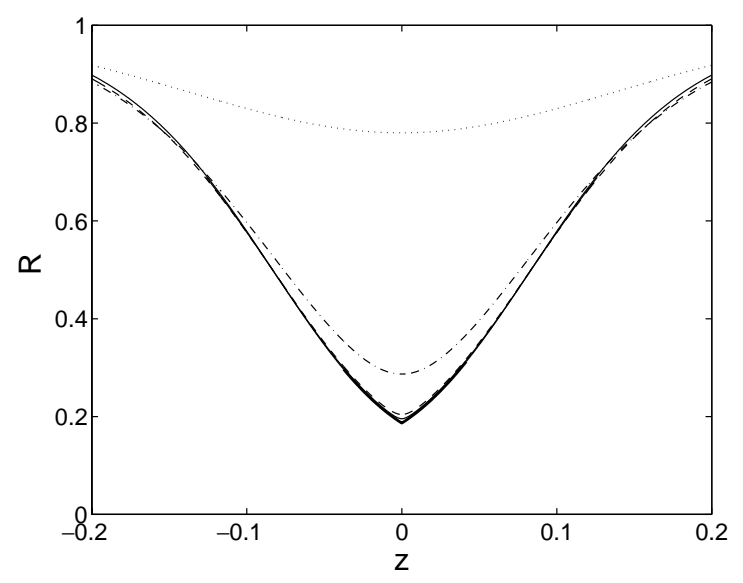

FIG. 2. $\quad R(z)$ as defined in Eqs. (13) versus $z$ (in units of $\tilde{\Lambda}^{-1}$ ), for $m=2$ (dotted line), 20 (dash-dotted), 100 (dashed), and 200 (solid). The thick solid line corresponds to the $m \rightarrow \infty$ solution given by Eq. (20). (i) Let us turn now to analyze large mass values, $m \gg \Lambda$. From Fig. 2 we see that there is a well-defined gaugino condensate profile in the $m \rightarrow \infty$ limit. In fact, if we assume that this limit exists, the following constraints should apply in the asymptotic regions:

$$
\begin{aligned}
& \rho(z) e^{i \alpha(z)}=R(z) e^{i \beta(z)} \quad(z \ll-1 / m), \\
& \rho(z) e^{i \alpha(z)}=R(z) e^{i[\beta(z)-2 \pi]} \quad(z \gg 1 / m) .
\end{aligned}
$$

In Fig. 3 we have drawn the combination $\frac{1}{2 \pi}[\beta(z)-$ $\alpha(z)$ ], confirming the previous statement; also a quick glance at Figs. 1 and 2 tells us that both $\rho$ and $R$ follow identical paths in the asymptotic regions.

Therefore, using Eq. (18) in this large $m$ limit we can get rid of $\alpha$ and $\rho$ in the BPS equations. Also the BPS constraint involves only the gaugino condensate and can be written as

$$
\operatorname{Im}\left\{e^{i[\gamma+\tilde{\beta}(z)]} R(z)\left[\ln \left(R(z) e^{i \tilde{\beta}(z)}\right)-1\right]\right\}=\mathrm{const},
$$

where $\tilde{\beta}(z)=\beta(z)$ for $z<0$ and $\tilde{\beta}(z)=\beta(z)-$ $2 \pi N_{f} / N_{c}$ for $z>0$. This constraint allows us to express $\beta$ as a function of $R$, and we end up with the following BPS equation for $R(z)$ :

$$
\begin{aligned}
\partial_{z} R(z)=6 N_{c}[R(z)]^{4 / 3} \tilde{\Lambda}\{\cos [\gamma+\tilde{\beta}(R(z))] \ln R(z) \\
-\sin [\gamma+\tilde{\beta}(R(z))] \tilde{\beta}[R(z)]\},
\end{aligned}
$$

together with the boundary condition at the origin calculated from Eq. (19), i.e.,

$$
R_{0}\left(1-\ln R_{0}\right)=\cos \left(\pi \frac{N_{f}}{N_{c}}\right) .
$$

As we can see from Fig. 4, this equation always has a solution with $R_{0}>1$. There is also a solution with $R_{0}<1$ when $2 N_{f}<N_{c}$. We have seen that only the case $R_{0}<1$ gives a finite, domain wall-like profile. We have also verified that the corresponding solution for $R(z)$

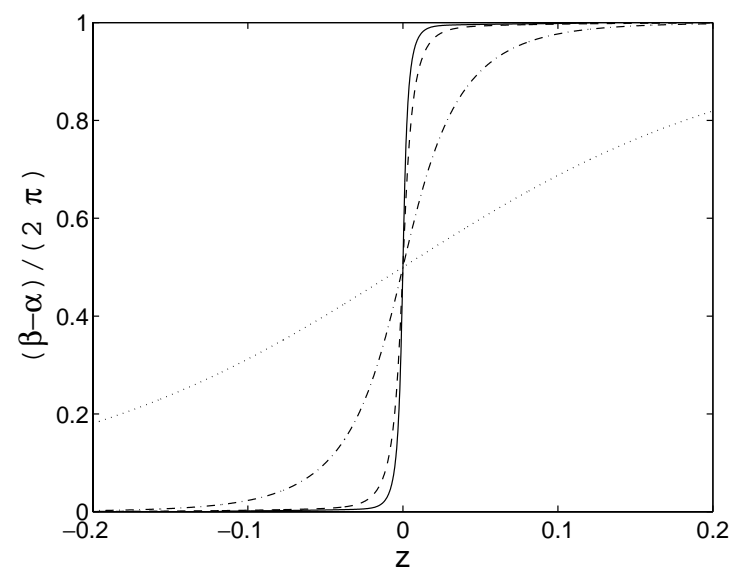

FIG. 3. Plot of the combination $\frac{1}{2 \pi}[\beta(z)-\alpha(z)]$ versus $z$ (in units of $\tilde{\Lambda}^{-1}$ ), for $m=2$ (dotted line), 20 (dash-dotted), 100 (dashed), and 200 (solid). 


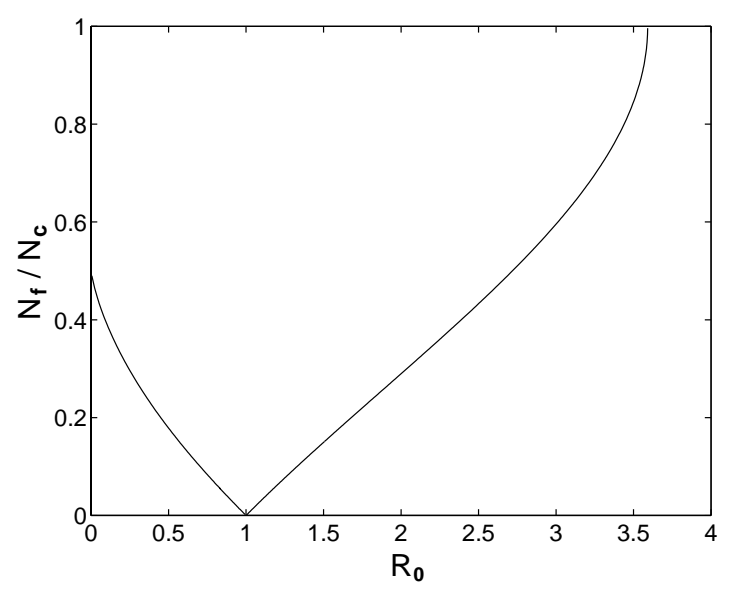

FIG. 4. Contour plot of the constraint Eq. (21) in the plane defined by the variables $R_{0}$ ( $x$ axis) and $N_{f} / N_{c}$ ( $y$ axis).

is precisely the large $m$ limit profile, which is represented by a thick solid line in Fig. 2 . Therefore the condition ensuring that there is just one branch of BPS states at low $m$ values also guarantees the existence of the large $m$ limit case.

In summary, it is possible to build BPS domain walls in SQCD with $2 N_{f}<N_{c}$, both in the weak coupling (Higgs) regime and in the strong coupling limit, where the theory approaches pure supersymmetric gluodynamics. When the Kähler metric is nonsingular along the different configurations the existence of these solutions in the strong coupling regime can be understood by just analyzing the superpotential, as in the cases we have just shown.

We thank A. Casas and M. Hindmarsh for useful discussions, and M. Seco for his invaluable help with the computers. The work of J.M.M. was supported by CICYT of Spain (Contract No. AEN98-0816). J. M. M. thanks the CERN Theory Division for hospitality, and we both acknowledge the British Council/Acciones
Integradas program for the financial support received through the Grant No. HB1997-0073.

*Email address: Beatriz.de.Carlos@cern.ch

†Email address: jesus@makoki.iem.csic.es

[1] A. Kovner and M. Shifman, Phys. Rev. D 56, 2396 (1997).

[2] G. Dvali and M. Shifman, Phys. Lett. B 396, 64 (1997); Phys. Lett. B 407, 452(E) (1997).

[3] A. Kovner, M. Shifman, and A. V. Smilga, Phys. Rev. D 56, 7978 (1997).

[4] B. Chibisov and M. Shifman, Phys. Rev. D 56, 7990 (1997); 58, 109901(E) (1998).

[5] M. Shifman, in The 6th International Symposium on Particles, Strings and Cosmology (PASCOS 98), Boston, MA, 1998 (to be published).

[6] G. Dvali, G. Gabadadze, and Z. Kakushadze, hep-th/ 9901032.

[7] E. Witten, Nucl. Phys. B507, 658 (1997).

[8] A. V. Smilga and A. I. Veselov, Phys. Rev. Lett. 79, 4529 (1997).

[9] A. V. Smilga and A. I. Veselov, Nucl. Phys. B515, 163 (1998).

[10] A. V. Smilga and A. I. Veselov, Phys. Lett. B 428, 303 (1998).

[11] A. V. Smilga, Phys. Rev. D 58, 065005 (1998).

[12] G. Veneziano and S. Yankielowicz, Phys. Lett. 113B, 231 (1982); T. Taylor, G. Veneziano, and S. Yankielowicz, Nucl. Phys. B218, 493 (1983).

[13] I. I. Kogan, A. Kovner, and M. Shifman, Phys. Rev. D 57, 5195 (1998).

[14] A. V. Smilga, in The 3rd Workshop on Continuous Advances in QCD (QCD 98), Minneapolis, MN, 1998 (to be published).

[15] B. de Carlos, M. Hindmarsh, N. McNair, and J.M. Moreno (to be published).

[16] V.S. Kaplunovsky, J. Sonnenschein, and S. Yankielowicz, Nucl. Phys. B522, 209 (1999). 